nearly. Consequently, light of wave-length greater than $\lambda 1090 \mathrm{~A}$. should not be able to excite the $\beta$-bands. Excitation can be brought about by $\lambda .1200 \mathrm{~A}$.; if $h \nu_{1}$ is taken to be in the neighbourhood of $\lambda 2750 \mathrm{~A}$. (Dutta), $h \nu_{2}$ comes out as near $\lambda 1250 \mathrm{~A}$.

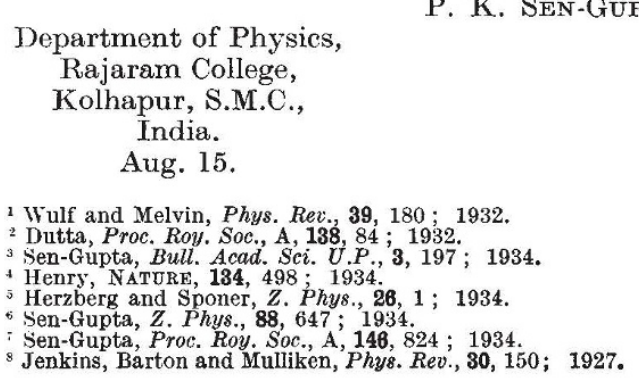

\section{Electron Diffraction by Vitreous Silica Powder}

I HAVE succeeded in showing recently that the comparatively coarse particles of any powder are suitable for structure investigation by means of electron diffraction ${ }^{1}$. For this purpose a mineral or other substance is ground carefully in a small mortar. The powder obtained is placed in a sedimentation air tube like that described by Gonell ${ }^{2}$. When blowing the slow jet of air through the heap of powder disposed at the bottom of this sedimentation tube, only the coarsest particles will return, while the minute ones will move with the air blast to the top of the tube where there is a big receiver. By placing there a suitable holder, for example, a loop of wire with a thin celluloid film for the transmission method, or a support for the reflection method, one can collect the particles, the sizes of which depend on the speed of the air blast. It is possible also to collect the smallest particles at the bottom of the tube. For this purpose it is necessary to blow the air for a few minutes only until the smoke appears at the top of the tube, and then to wait, in the case of a tube of one metre in length, in general about 10-15 minutes. Then all particles larger than $0 \cdot 1-1 \cdot 0 \mu$ in size are precipitated at the bottom of the tube, the remaining being still suspended in the air. To collect the latter on the holder it is sufficient to wait in the case mentioned above about half an hour, after which the process had to be repeated a few times to accumulate a layer of sufficient thickness. It may be noted that the diffraction haloes from the celluloid film disappear completely only when the layer is thick enough. In this way I have obtained sometimes sufficiently clear diffraction patterns from specimens, which were otherwise quite unsuitable for electron diffraction, for example, ignited magnesia, sand, cements and tripolites. Mrs. L. I. Tatarinowa has proposed another method for preparing the specimens by drying on the celluloid film a drop of liquid with a fine suspension of the substance to be studied. This method, which gives sometimes excellent specimens for electron diffraction, has an especial advantage in that it requires only the minutest portion of substance. I have used successfully both these methods.

Here I will confine myself to the case of electron diffraction by vitreous silica glass, which does not give sharp rings when exposed to X-rays. It is of interest that such an amorphous body behaves like a true crystalline aggregate (see Fig. 1). On the best photographic plates, I have succeeded in measuring sometimes up to ten and even more distinct rings, which correspond approximately to the following Bragg spacings $d$ :

\begin{tabular}{|c|c|c|c|}
\hline Int. & $h k l l$ & (observed) & (calculated) \\
\hline Weak & - & $4 \cdot 40$ & - \\
\hline Very strong & 111 & $4 \cdot 03$ & $4 \cdot 04$ \\
\hline Strong & 002 & $3 \cdot 64$ & $3 \cdot 64$ \\
\hline Very strong & 022 & $2 \cdot 52$ & $2 \cdot 50$ \\
\hline Weak & 113 & $2 \cdot 17$ & $2 \cdot 17$ \\
\hline ", & 222 & 1.99 & $2 \cdot 02$ \\
\hline & 133 & $1 \cdot 65$ & $1 \cdot 62$ \\
\hline Fair & 224 & $1 \cdot 47$ & $1 \cdot 46$ \\
\hline Weak & 242 & $1 \cdot 42$ & $1 \cdot 42$ \\
\hline Fair & 044 & $1 \cdot 26$ & $1 \cdot 25$ \\
\hline Very weak & 026 & $1 \cdot 11$ & $1 \cdot 14$ \\
\hline$" \quad "$ & 046 & 1.01 & 0.99 \\
\hline
\end{tabular}

(The first extra ring belongs presumably to an adsorbed organic substance from the vacuum grease.) Some of these rings, namely, the most intense ones, have been observed by L. R. Maxwell and V. M. Mosley $^{3}$ in their work on electron diffraction by transmission of electrons through thin films of silica glass. In spite of the lack of accuracy of the measure-

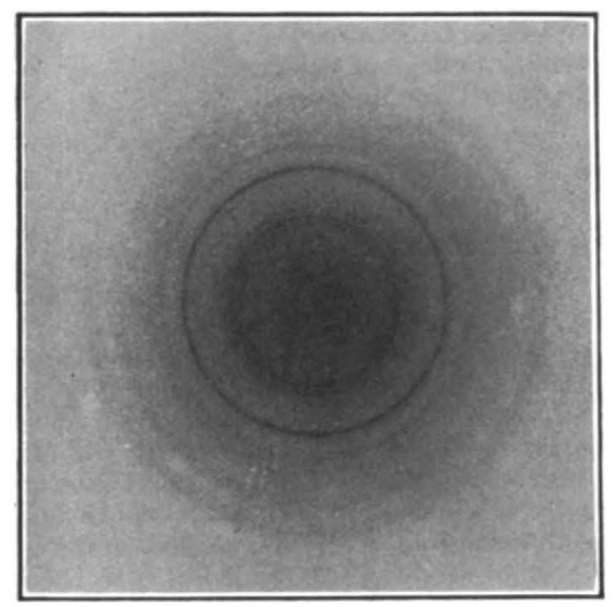

Fig. 1. Electron diffraction pattern of vitreous silica.

$$
\times 2 \text {. }
$$

ments, I am inclined to think that the above results can only be interpreted as a scattering by tetragonal cristobalite crystallites constituting the vitreous $\mathrm{SiO}_{2}$. The axial ratio is $1.06 ; a=6 \cdot 87 ; c=7 \cdot 28$. The size of these cristallites, which is probably of the order of 15-20 A., as estimated by J. T. Randall, H. P. Rooksby and B. S. Cooper ${ }^{4}$ by measuring the breadth of X-ray diffraction band, is sufficient to give very sharp diffraction rings. The electron wavelengths in my experiments were about $0.06 \mathrm{~A}$., while these authors, as well as B. E. Warren ${ }^{5}$, used an $\mathrm{X}$-ray wave-length of $1.54 \mathrm{~A}$., so that the sharp electron diffraction patterns with many lines are, of course, the consequence of greater resolving power of the electron rays. Hence the method of electron diffraction is applicable to a much larger range of substances than hitherto supposed.

A fuller account is being published elsewhere. ${ }^{6}$

$$
\begin{gathered}
\text { Cement Institute, } \\
\text { Leningrad, } 53 .
\end{gathered}
$$$$
\text { N. A. Shishacow. }
$$

${ }^{2}$ C. R. Acad. Sci. U.S.S.R., No, 7-8, 461, March 1935. 2 Tonindustrie Z., No. 13,$243 ; 1929$.

3 Phys. Rev., 47, 331; 1935.

4 Z. Krist., 75, 196; ; 1935

s P. Krist., 75, 196; 1930.

- Z. techn. Phys. (Russian). 